\title{
Swarm-Based Multi-Objective Design Optimization of Single-Plate Condenser MEMS Microphone
}

\author{
Qummar Zaman 1 , Senan Alraho', Andreas König ${ }^{1}$ \\ ${ }^{1}$ Lehrstuhl Integrierte Sensorsysteme (ISE), TU Kaiserslautern Deutschland, \\ qummar@eit.uni-kl.de
}

\begin{abstract}
Summary:
This work presents a multi-objective optimization of MEMS microphone using the PolyMUMPS fabrication technology. The proposed method handles simultaneous optimization of performance parameters such as sensitivity, lower cut-off frequency, resonance frequency, linearity and noise level. An agglomerative method is being used for multi-objective optimization due to its simplicity. While for efficient exploration of complex search space of MEMS microphone, the traditional particle swarm optimizer has been amended by adaptively adjusting its social and personal best coefficients.
\end{abstract}

Keywords: MEMS condenser microphone, Machine learning for microsystems, An agglomerative method, Particle swarm optimizer, Multi-objective optimization, PolyMUMPS technology

\section{Background, Motivation an Objective}

A microelectromechanical system (MEMS) based condenser microphone also known as silicon microphone [1] is widely used as an acoustic wave (sound, pressure waves, etc.) sensor [2]. The applications of a MEMS microphone include hearing aids, vibration, security surveillance instruments, voice control devices and machine conditioned monitoring in industry 4.0 $[3,4]$. The main reasons for this trend are their small size, compatibility with the standard CMOS process, low noise level, low power consumption, flat frequency response and high sensitivity [5].

Several parameters (sensitivity, lower cut-off frequency, resonance frequency, linearity and noise level) affect the performance of MEMSbased microphones [6]. Hence, multiple objectives need to be explored simultaneously. So, the main objective of this research is to analyze the multi-objective performance optimization of a MEMS-based microphone. Many researchers have worked on the optimizations of the MEMS microphone [1] [6-10] but none of them covered all the optimization parameters at the same time. MEMS design implies a search space complexity, that makes it challenging to find the suitable optimum using traditional optimization algorithms [8]. To handle this problem, metaheuristic and evolutionary algorithms are found promising alternatives for the optimization of such a complex landscape. Additionally, they showed promising results in many engineering optimization problems. For this reason, Particle Swarm Optimizer (PSO) is selected as an evolutionary optimizer for this research. The multi-objective optimization of a microphone is presented in [8] that simultaneously considers the trade-off between noise floor and sensitivity. Similarly, [1] used evolutionary genetic algorithm to optimize three objective functions for MEMS microphone but did not consider linearity and noise level optimization.

\section{Proposed Methodology}

The proposed work presents a novel PSO method to optimize a microphone due to its enhanced exploration capabilities through adaptive value adjustment of personal and social coefficients presented in [11]. PSO begins with random initialization followed by the evolution of cost function. Then the particle's personal or global best is being amended in case of better fitness value. The cognitive and social scaling factors are being updated according to the following equations

$$
c_{1}=c_{2}=F(D)=\frac{a}{1+e^{-c(D-d)}}+b
$$

where $a=0.5, b=1.5, c=0.000035 \times$ search range (distance between upper \& lower bound of particle), $d=0$ and $D=P_{p \text { or } g}(k)-x_{i}(k)$ which represent the distances of the particle to its pest or gbest at kth iteration. After that, the particle's velocity and position are being updated, this procedure continues until maximum iterations.

Also, we applied an agglomerative method for multi-objective optimization [12] of a microphone. The weight parameters of all optimization 
objective for an agglomerative method are defined equally. The 3D structure of a microphone is designed in coventor mems+ [13] using PolyMUMPS technology as shown in Fig. 1.

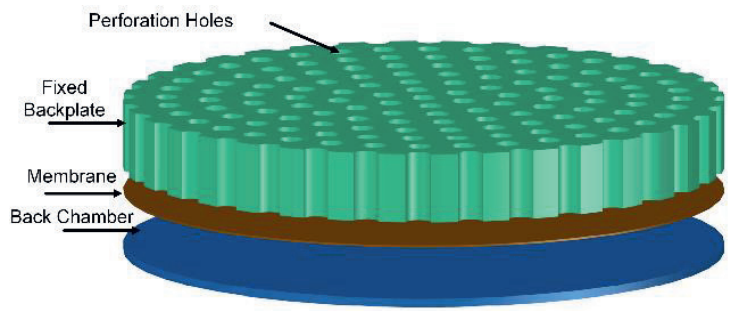

Fig. 1. A $3 D$ structure of condenser MEMS microphone.

\section{Results}

The design specification for MEMS microphone and results achieved after optimization along with search variables' range and step size are presented in Tables 1 and 2 respectively. For this experiment we used 10 particles and 100 number of iterations.

Tab. 1: Design specifications for MEMS microphone.

\begin{tabular}{|l|c|c|}
\hline \multicolumn{1}{|c|}{ Specifications } & Target design & Achieved \\
\hline Sensitivity & $\leq 5 \mathrm{fF}(\mathrm{Pa})^{-1}$ & $\geq 5.34 \mathrm{fF}(\mathrm{Pa})^{-1}$ \\
\hline $\begin{array}{l}\text {-3dB low } \\
\left.\text { frequency ( } \mathrm{f}_{1}\right)\end{array}$ & $\geq 20 \mathrm{kHz}$ & $3.433 \mathrm{~Hz}$ \\
\hline $\begin{array}{l}\text { Resonance } \\
\left.\text { frequency ( } \mathrm{f}_{2}\right)\end{array}$ & $\geq 1 \mathrm{pF}$ & $59.71 \mathrm{kHz}$ \\
\hline DC capacitor & 1 to $20 \mathrm{~Pa}$ & $1 \mathrm{to} 20 \mathrm{~Pa}$ \\
\hline Linearity & $\leq 40 \mathrm{pV}(\mathrm{Hz})^{-1 / 2}$ & $37.11 \mathrm{pV}(\mathrm{Hz})^{-1 / 2}$ \\
\hline Noise level &
\end{tabular}

Tab. 2: Range of search space variables.

\begin{tabular}{|l|c|c|c|}
\hline \multirow{2}{*}{ Design Variables } & \multicolumn{3}{|c|}{ Range } \\
\cline { 2 - 4 } & From & To & $\begin{array}{c}\text { Step } \\
\text { Size }\end{array}$ \\
\hline Membrane thickness & $0.5 \mu \mathrm{m}$ & $5 \mu \mathrm{m}$ & $100 \mathrm{~nm}$ \\
\hline Membrane Radius & $500 \mu \mathrm{m}$ & $750 \mu \mathrm{m}$ & $100 \mathrm{~nm}$ \\
\hline Backplate thickness & $2.5 \mu \mathrm{m}$ & $8 \mu \mathrm{m}$ & $100 \mathrm{~nm}$ \\
\hline $\begin{array}{l}\text { Backplate conductive } \\
\text { factor }\end{array}$ & 0.5 & 0.75 & 0.05 \\
\hline Air gap thickness & $1 \mu \mathrm{m}$ & $8 \mu \mathrm{m}$ & $100 \mathrm{~nm}$ \\
\hline $\begin{array}{l}\text { Perforation hole } \\
\text { radius }\end{array}$ & $2.5 \mu \mathrm{m}$ & $25 \mu \mathrm{m}$ & $100 \mathrm{~nm}$ \\
\hline Perforation distance & $2.5 \mu \mathrm{m}$ & $25 \mu \mathrm{m}$ & $100 \mathrm{~nm}$ \\
\hline Ventilation radius & $1 \mu \mathrm{m}$ & $5 \mu \mathrm{m}$ & $100 \mathrm{~nm}$ \\
\hline
\end{tabular}

The output sensitivity graph is shown in Fig. 2 which clearly illustrates the flattened response of sensitivity and makes it perfectly applicable for audio frequency applications. The outlook to this work will be the introduction of self-x (self-calibration, self-healing) properties to the MEMS microphone [14] along with its electronic readout circuit to address the problem of device performance tolerances or drift due to static and dynamic effects.

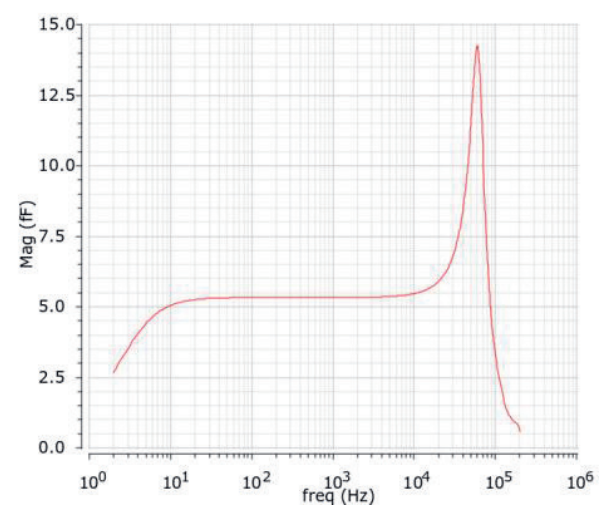

Fig. 2. Frequency response of output sensitivity.

\section{References}

[1] G. M. Sessler, "Silicon microphones," J. Audio Eng. Soc. 44, 16-22 (1996).

[2] M. Saadatmand and J. Kook, "Multi-objective optimization of a circular dual back-plate MEMS microphone: tradeoff between pull-in voltage, sensitivity and resonance frequency," Microsyst. Technol. 2019.

[3] S. Babaei Sedaghat, B. Azizolla Ganji, and R. Ansari, "Design and modeling of a frog-shape MEMS capacitive microphone using SOI technology," Microsyst. Technol., vol. 24, Jun. 2017.

[4] Shaffer, Derek, et al. "Investigation of the feasibility of using microphone arrays in monitoring machining conditions." Manufacturing Letters 15 (2018).

[5] M. A. Shah, I. A. Shah, D. G. Lee, and S. Hur, "Design approaches of MEMS microphones for enhanced performance," J. Sensors, vol. 2019, 2019.

[6] Farnsworth, Michael, et al. "An efficient evolutionary multi-objective framework for MEMS design optimisation: validation, comparison and analysis." Memetic Computing 3.3 (2011): 175.

[7] Wang, Pan, Qibing Lu, and Zhun Fan. "Evolutionary design optimization of MEMS: a review of its history and state-of-the-art." Cluster Computing 22.4 (2019): 9105-9111." IEEE Sensors Journal 19.15 (2019).

[8] Papila, Melih, et al. "Piezoresistive microphone design pareto optimization: tradeoff between sensitivity and noise floor." Journal of MEMS 15.6 (2006).

[9] Shah, Muhammad Ali, et al. "Design approaches of MEMS microphones for enhanced performance." Journal of Sensors 2019 (2019)

[10] Zhu, Jianxiong, et al. "Development Trends and Perspectives of Future Sensors and MEMS/NEMS." Micromachines 11.1 (2020): 7.

[11] Liu, Weibo, et al. "A Novel Sigmoid-Function-Based Adaptive Weighted Particle Swarm Optimizer." IEEE transactions on cybernetics (2019).

[12] Kammara, Abhaya Chandra, Lingaselvan Palanichamy, and Andreas König. "Multi-objective optimization and visualization for analog design automation." Complex \& Intelligent Systems 2.4 (2016): 251-267.

[13] "MEMS Microphone Design - Coventor MEMSplus." https://www.coventor.com/mems-solutions/microphones/. Accessed: 26-11-2020.

[14] Clark, Jason. "Self-Calibration and Performance Control of MEMS with Applications for IoT." Sensors 18.12 (2018): 4411. 